\title{
OCORRÊNCIA DE MORDIDA ABERTA ANTERIOR E HÁBITOS BUCAIS DELETÉRIOS EM CRIANÇAS DE 4 A 6 ANOS
}

\author{
Occurrence of anterior open bite and harmful oral habits \\ in children from 4 to 6-year old
}

\author{
Maritza Zapata ${ }^{(1)}$, Joanna Carolina Bachiega ${ }^{(2)}$, Analúcia Ferreira Marangoni ${ }^{(3)}$, \\ José Eduardo Massucato Jeremias ${ }^{(4)}$, Raquel Agnelli Mesquita Ferrari ${ }^{(5)}$, \\ Sandra Kalil Bussadori (6), Elaine Marcílio Santos ${ }^{(7)}$
}

\begin{abstract}
RESUMO
Objetivo: verificar a ocorrência de mordida aberta anterior e de hábitos orais deletérios em crianças de quatro a seis anos de idade. Métodos: foram avaliadas 266 crianças de quatro a seis anos incompletos, de ambos os gêneros, na cidade de Suzano - São Paulo. As avaliações constaram de aplicação de ficha de avaliação para detecção de ocorrência de hábitos bucais deletérios aos responsáveis e da realização de exame clínico constituído por avaliação da oclusão das crianças. Resultados: verificou-se que 221 crianças $(83,1 \%)$ apresentaram pelo menos um hábito bucal deletério, sendo o mais frequente o uso de mamadeira 167 (75,6\%). A ocorrência simultânea de hábitos deletérios e alterações de oclusão dentária foi observada em 119 crianças $(44,1 \%$ da amostra) e nestas, a maior prevalência foi a presença da mordida aberta anterior, presente em 89 (79,8\%). Conclusão: a mordida aberta anterior (MAA) foi a alteração oclusal mais prevalente nas crianças, havendo associação estatisticamente significante entre hábitos orais deletérios, como uso de mamadeira, chupeta e ocorrência de bruxismo e presença de mordida aberta anterior.
\end{abstract}

DESCRITORES: Oclusão Dentária; Hábitos; Mordida Aberta

(1) Dentista; Consultório Maritza Zapata, Suzano, SP; Especialista em Odontopediatria pela Universidade de Mogi das Cruzes.

(2) Dentista; Especialista em Odontopediatria; Mestranda em Ciências da Reabilitação pela Universidade Nove de Julho.

(3) Dentista; Marangoni Odontologia, SP; Especialista em Odontopediatria e Ortopedia Funcional dos Maxilares; Mestranda em Ciências da Reabilitação pela Universidade Nove de Julho.

(4) Fisioterapeuta; Prefeitura Municipal de São Sebastião, SP; Mestrando em Ciências da Reabilitação pela Universidade Nove de Julho.

(5) Fisioterapeuta; Professora do Programa de Mestrado em Ciências da Reabilitação da Universidade Nove de Julho, São Paulo, SP; Mestre e Doutora em Ciências Fisiológicas pela Universidade Federal de São Carlos.

(6) Dentista; Professora do Programa de Mestrado em Ciências da Reabilitação da Universidade Nove de Julho, São Paulo, SP; Mestre em Odontologia (Materiais Dentários) pela Universidade de São Paulo; Doutora em Ciências Odontológicas pela Universidade de São Paulo.

(7) Dentista; Professora Titular da Disciplina de Odontopediatria da Universidade Camilo Castelo Branco, São Paulo, SP; Mestre em Odontologia (Patologia Bucal e Ortopedia Funcional dos Maxilares) pela Universidade de São Paulo; Doutora em Odontopediatria pela Universidade de São Paulo.

Conflito de interesses: inexistente

\section{INTRODUÇÃO}

Durante o primeiro ano de vida, a boca é a região mais importante do corpo, e a sucção uma resposta natural da própria espécie, considerada um dos primeiros padrões comportamentais do indivíduo ${ }^{1}$. No entanto, a função muscular alterada, induzida por hábitos de sucção não nutritivos, correlacionase com a mordida aberta anterior, levando a uma alteração na deglutição normal e deformação da arcada dentária e palato ${ }^{2-4}$.

As mordidas abertas anteriores (MAA) são desarmonias oclusais geralmente associadas a hábitos bucais anormais, que são comportamentos adquiridos e que, em função da repetição contínua, automatizam-se e aperfeiçoam-se, tornando-se assim inconscientes ${ }^{5}$.

Se estes hábitos bucais persistirem durante 0 período da dentição mista, após o surto de crescimento, é provável que venham a provocar maloclusões, comprometendo ainda mais a deglutição e somando-se aos fatores ambientais, como 
hipertrofia de amígdalas, respiração bucal e deficiências fonoarticulatórias ${ }^{6-9}$. Estudos relatam que crianças que abandonam o hábito deletério até os seis anos de idade apresentam correção espontânea das maloclusões associadas à MAA 10-12.

Quando a criança tem amamentação natural ela suga o alimento, o que Ihe traz prazer oral e satisfaz sua fome, além de exercitar sua musculatura. Já uma criança que recebe o alimento por mamadeiras apresenta tendência para colocar o dedo na boca e, como o bico da mamadeira permite um maior fluxo de saída de leite, é nesse momento que sugar se torna um hábito nocivo, podendo ocasionar alterações na forma do arco e profundidade do palato ${ }^{13,14}$.

O uso de mamadeiras e chupetas faz com que o bebê não seja devidamente estimulado na área sensório-motora, podendo se desinteressar pela sucção do leite materno. A partir deste momento, a musculatura perioral e da língua podem tornar-se hipotônicas, levando a uma alteração na deglutição normal, ocasionando mordida aberta anterior ou posterior ${ }^{15}$.

Os hábitos bucais deletérios constituem motivos para estudos multidisciplinares em relação às suas origens e consequências nos meios médico, psicológico, fonoaudiológico e odontológico, a fim de que os resultados adquiridos sejam mais rápidos, efetivos e estáveis ${ }^{16-18}$. Os fatores ambientais (hábitos) são determinantes na incidência da maloclusão em idade precoce, assim como os problemas de ordem genética o são em idades mais avançadas ${ }^{19}$. Os distúrbios funcionais podem ser considerados como agentes etiológicos primários ou secundários, além de poder corroborar a predisposição a desordens temporomandibulares ${ }^{20}$.

Diante das considerações anteriores, o presente estudo objetivou verificar a ocorrência da mordida aberta anterior e sua relação com a presença de hábitos bucais deletérios em crianças.

\section{MÉTODOS}

Trata-se de um estudo transversal, descritivo, elaborado de acordo com as diretrizes da resolução 196/96 do Conselho Nacional de Saúde e realizada mediante a autorização da direção da escola onde foi executada. Os responsáveis pelas crianças foram devidamente informados quanto à metodologia utilizada e, após concordância, assinaram Termo de Consentimento Livre e Esclarecido.

Foram assumidos como critérios de inclusão todos os responsáveis pelos voluntários que leram e assinaram o termo de consentimento livre e esclarecido e crianças na faixa etária de quatro a seis anos. Como critérios de exclusão, crianças portadoras de dentes com anomalias de forma e tamanho e já submetidas ou em tratamento ortodôntico.

Foram avaliadas 266 crianças, de ambos os gêneros, com idade de quatro a seis anos que frequentavam a escola Toshio Utyama, do Município de Suzano, SP. A avaliação foi iniciada pelo preenchimento de fichas de avaliação pelos responsáveis, relacionando à presença ou não de hábitos parafuncionais (mamadeira, chupeta, onicofagia, bruxismo, interposição ou sucção de objeto ou dedo), em relação ao seu tipo e frequência, aplicadas pelo cirurgião-dentista responsável.

$\mathrm{Na}$ sequência, as crianças foram submetidas à avaliação clínica pelo próprio pesquisador (cirurgião-dentista), na qual foram utilizadas espátulas de madeira e luz natural para a observação da presença ou não de maloclusões. O diagnóstico de oclusão foi sempre obtido em relação cêntrica $(R C)$.

Esta pesquisa foi aprovada pelo Comitê de Ética em Pesquisa da Universidade de Mogi das Cruzes, com protocolo no 174/2005.

Os dados foram analisados com o auxílio do software Statistical Package for Social Sciences (SPSS@ for Windows 10.0, SPSS Inc., Chicago, IL). A ocorrência dos hábitos orais deletérios mamadeira, chupeta, onicofagia, bruxismo, interposição de objetos e dedo foi comparada entre as crianças que apresentavam ou não alterações oclusais, utilizando-se o teste do qui-quadrado de Pearson e o teste exato de Fisher. Valores de $p$ menores que 0,05 foram considerados estatisticamente significantes.

\section{RESULTADOS}

Das 266 crianças, cujos responsáveis responderam aos questionários, $132(49,6 \%)$ eram do gênero masculino e $134(51,4 \%)$ do feminino. A idade média foi de 4,7 anos com desvio padrão de 0,6 . Em relação à cor da pele $200(75,2 \%)$ crianças foram classificadas como leucodermas, 34 (12,8\%) melanodermas e 32 (12\%) como xantodermas. Com relação à dentição, $174(65,4 \%)$ crianças apresentavam dentição exclusivamente decídua, enquanto $92(34,6 \%)$ apresentavam dentição mista.

Apenas $45(16,9 \%)$ das 266 crianças avaliadas não apresentavam hábitos orais deletérios, enquanto $221(83,1 \%)$ apresentavam pelo menos um hábito, sendo que o uso da mamadeira foi o mais comum, presente em 167 (75,6\%) seguido por chupeta $93(42,1 \%)$ e onicofagia $52(23,52 \%)$. A ocorrência dos demais hábitos orais deletérios está demonstrada na Tabela 1.

A presença simultânea de dois ou mais hábitos deletérios foi muito comum, sendo verificada em 134 crianças $(60,63 \%)$ das 221 crianças que 
Tabela 1 - Hábitos orais deletérios apresentados pelas crianças avaliadas

\begin{tabular}{lc}
\hline Hábito deletério & $\begin{array}{c}\text { Ocorrência } \\
\mathbf{N}=\mathbf{2 2 1}(\%)\end{array}$ \\
\hline Mamadeira & $167(75,6 \%)$ \\
Chupeta & $93(42,1 \%)$ \\
Onicofagia & $52(23,5 \%)$ \\
Bruxismo & $48(21,1 \%)$ \\
Objeto & $31(14 \%)$ \\
Dedo & $12(5,4 \%)$ \\
\hline
\end{tabular}

apresentavam hábitos deletérios. A associação mais encontrada foi a de mamadeira e chupeta -72 crianças $(53,73 \%)$, seguida de mamadeira e onicofagia (36 crianças, 26,8\%), mamadeira e bruxismo (31 crianças, 23,13\%), mamadeira, chupeta e bruxismo (17 crianças, $12,6 \%$ ) e mamadeira e interposição de objetos (14 crianças, $10,44 \%$ ), como ilustrada na Tabela 2.

Tabela 2 - Frequência de associações de hábitos orais deletérios nas crianças avaliadas

\begin{tabular}{|c|c|}
\hline $\begin{array}{l}\text { Associação de hábitos deletérios } \\
(n=134)\end{array}$ & \\
\hline Mamadeira/chupeta & $72(53,3 \%)$ \\
\hline Mamadeira/onicofagia & $36(26,8 \%)$ \\
\hline Mamadeira/bruxismo & $31(23,13 \%)$ \\
\hline Mamadeira/chupeta/bruxismo & $17(12,6 \%)$ \\
\hline Mamadeira/interposição de objetos & $14(10,44 \%)$ \\
\hline
\end{tabular}

Alterações de oclusão dentária foram detectadas em 119 (44,7\%) das 266 crianças avaliadas. Aplicou-se teste Qui-quadrado com correlação de Pearson e teste Exato de Fisher e constatou-se que a mordida aberta anterior foi a alteração mais frequente estando presente em $89(74,8 \%)$ das 119 crianças que apresentaram alguma alteração de oclusão, sendo que a mordida aberta posterior foi encontrada em apenas um caso $(0,8 \%)$ e a mordida cruzada anterior esteve presente em seis $(5,0 \%)$ e a posterior em 18 crianças $(15,2 \%)$, considerando que 5 crianças $(4,2 \%)$ apresentaram associação das mordidas cruzada anterior e posterior, conforme evidenciado na Tabela 3.

Em relação à presença de hábitos deletérios $e$ mordida aberta anterior, das 89 crianças com essa alteração oclusal, $30(33,8 \%)$ tinham o hábito de tomar mamadeira, $25(28,1 \%)$ de chupar chupeta e $11(12,3 \%)$ tinham bruxismo. Houve associação estatisticamente significante entre a MAA e os hábitos mamadeira $(p=0,006)$, chupeta $(p<0,001)$ e bruxismo $(p=0,03)$, conforme resultados descritos na Tabela 4.

Tabela 3 - Prevalência de alterações de oclusão nas 119 das 266 crianças avaliadas

\begin{tabular}{lc}
\hline Alteração de Oclusão & $\begin{array}{c}\text { Prevalência } \\
\mathbf{N}=\mathbf{1 1 9}\end{array}$ \\
\hline Mordida Aberta & \\
Anterior & $89(74,8 \%)$ \\
Posterior & $1(0,8 \%)$ \\
Mordida Cruzada & \\
Anterior & $6(5,0 \%)$ \\
Posterior & $18(15,2 \%)$ \\
Anterior e Posterior & $5(4,2 \%)$ \\
\hline
\end{tabular}

Tabela 4 - Ocorrência dos hábitos orais deletérios de acordo com a presença ou não de mordida aberta anterior das 266 crianças avaliadas

\begin{tabular}{lccc}
\hline \multirow{2}{*}{ Hábito deletério } & \multicolumn{2}{c}{ Mordida Aberta Anterior } & \multirow{2}{*}{$\mathbf{p}^{\dagger}$} \\
\cline { 2 - 3 } & \multicolumn{1}{c}{$\operatorname{Sim}(\mathbf{n}=\mathbf{8 9})$} & $\mathbf{N a ̃ o}(\mathbf{n}=\mathbf{1 7 7})$ & $\mathbf{0 , 0 0 6}$ \\
Mamadeira & $30(33,8 \%)$ & $93(52,5 \%)$ & $<\mathbf{0 , 0 0 1}$ \\
Chupeta & $25(28,1 \%)$ & $27(15,2 \%)$ & 0,9 \\
Onicofagia & $8(9,0 \%)$ & $36(20,3 \%)$ & $\mathbf{0 , 0 3}$ \\
Bruxismo & $11(12,3 \%)$ & $25(14,1 \%)$ & 0,6 \\
Objeto & $4(4,5 \%)$ & $24(13,5 \%)$ & 0,2 \\
Dedo & $3(3,4 \%)$ & $7(4,0 \%)$ & \\
\hline
\end{tabular}




\section{DISCUSSÃO}

Os resultados do presente estudo evidenciaram que a mordida aberta anterior (MAA) foi a alteração oclusal mais prevalente nas crianças avaliadas. Além disso, foi observada associação estatisticamente significante entre mamadeira, chupeta ou bruxismo e a presença de mordida aberta anterior, sugerindo que estes hábitos orais deletérios estejam relacionados a este tipo de maloclusão ${ }^{14,15,20}$.

Os principais fatores causadores de mordida aberta anterior são os hábitos de sucção digital ou de chupeta, mamadeira, deglutição atípica e interposição de língua ou lábio, podendo também corroborar as disfunções temporomandibulares ${ }^{7,20,21}$.

A mordida aberta anterior esteve presente em 89 crianças, sendo estes dados semelhantes aos encontrados em estudo anterior ${ }^{22}$, onde foram avaliadas 904 crianças de três a seis anos, com esta alteração oclusal em $27,6 \%$ da amostra.

Quanto aos hábitos deletérios mais comumente relacionados à desarmonia oclusal, os encontrados neste estudo reafirmam estudos realizados anteriormente ${ }^{23,24}$.

A remoção de hábitos de sucção em crianças em fase de dentição decídua pode proporcionar a correção ou atenuação de maloclusão do tipo mordida aberta anterior, a adequação de estruturas e o redirecionamento de funções do sistema estomatognático. Porém, somente a remoção dos hábitos pode não promover total adequação destas funções, sendo assim, a utilização da Terapia Miofuncional Orofacial pode favorecer o aumento da força muscular, provocar mudanças positivas nos padrões funcionais, e assim prevenir desvios no desenvolvimento craniofacial ${ }^{12,25-27}$.
A ocorrência da associação de mais de um hábito oral deletério foi muito comum ${ }^{9}$, o também foi evidenciado em estudo anterior ${ }^{28}$.

Com base nos resultados encontrados torna-se evidente a necessidade de uma abordagem multidisciplinar para detecção, prevenção e intervenção precoce no que se refere à presença de hábitos orais deletérios, de forma a minimizar a ocorrência de alterações oclusais, uma vez que o tratamento em fases tardias é mais complexo, envolvendo, em alguns casos, a cirurgia ortognática ${ }^{7,20}$. Além disso, no diagnóstico da mordida aberta anterior, o conhecimento do fator etiológico é fundamental para que se possa atuar de maneira objetiva, eliminando os fatores predisponentes com o tratamento adequado, permitindo assim que haja crescimento e desenvolvimento faciais normais, sem o estabelecimento de maloclusões na dentadura permanente ${ }^{5}$.

Este tipo de abordagem em relação à mordida aberta anterior faz observar a necessidade de mais estudos, tanto em relação à incidência e prevalência da mesma, quanto aos métodos que podem ser mais eficazes no restabelecimento da oclusão do paciente, para que haja um pronto restabelecimento da função estomatognática do paciente.

\section{CONCLUSÃO}

A mordida aberta anterior (MAA) foi a alteração oclusal mais prevalente nas crianças. Houve associação estatisticamente significante entre hábitos deletérios a presença de mordida aberta anterior, sugerindo que este tipo de maloclusão possa estar associado aos hábitos orais deletérios.

\section{ABSTRACT \\ Purpose: to determine the occurrence of anterior open bite and harmful oral habits in children from four to six-year old. Methods: a total of 266 four-to-six-year-old male and female children in the city of Suzano (São Paulo, Brazil) were evaluated. An assessment chart for detecting the occurrence of harmful oral habits was administered to the guardians and clinical exams were performed for assessing occlusion in the children. Results: a total of 221 children $(83.1 \%)$ had at least one harmful oral habit, the most frequent of which was the use of a feeding bottle $(n=167 ; 75.6 \%)$. The occurrence of simultaneous harmful habits and dental occlusion abnormalities was observed in 119 children (44.1\% of the sample); among these children, the greatest prevalence was of anterior open bite $(n=89$; $79.8 \%$ ). Conclusion: anterior open bite was the most prevalent occlusion alteration in the children and had a statistically significant association with harmful oral habits, such as the use of a bottle and/ or pacifier and teeth grinding.}

KEYWORDS: Dental Occlusion; Habits; Open Bite 


\section{REFERÊNCIAS}

1. Corrêa MSNP. Odontopediatria na primeira infância. São Paulo: Santos; 2003. 970p.

2. Zuanon ACC, Oliveira MF, Giro EMA, Maia JP. Influência da amamentação natural e artificial no desenvolvimento de hábitos bucais. J Bras Odontopediatr Odontol Bebê. 2000; 2(8):303-6.

3. Alió-Sanz JJ. A new cephalometric diagnostic method for Down's Syndrome patients with open bite. Med Oral Patol Oral Cir Bucal. 2008 Mar; 13(3):E171-5.

4. Farias AC, Carnasciali MCG. Aleitamento materno e hábitos nocivos e a sua relação com as alterações oclusais. Rev Dens. 2006; 14(2):79.

5. Arat ZM, Akcam MO, Esenlik E, Arat FE. Inconsistencies in the differential diagnosis of open bite. Angle Orthod. 2008 May; 78(3):415-20.

6. Sodré AS, Franco EA, Monteiro DF. Mordida aberta anterior. J Bras Ortodon Ortop Facial. 1998; 3(17):80-4.

7. Bicalho GP, Motta ARM, Vicente LCC. Avaliação da deglutição em crianças respiradoras orais. Rev CEFAC. 2006; 8(1):50-5.

8. Maciel CTV, Leite ICG. [Etiological aspects of anterior open bite and its implications to the oral functions]. Pró-Fono. 2005; 17(3):293-302.

9. Amary ICM, Rossi LAF, Yumoto VA, AssencioFerreira VJ, Marchesan IQ. Hábitos deletériosalterações de oclusão. Rev CEFAC. 2002; 4(1):123-6.

10. Bertoldi PM, Felício CM, Matsumoto MA. [Effect of the early intervention of oral habits on the development of dental occlusion]. Pró-Fono. 2005 Jan-Ap; 17(1):37-44.

11. Trawitzki LV, Anselmo-Lima WT, Melchior MO, Grechi TH, Valera FC. Breast-feeding and deleterious oral habits in mouth and nose breathers. Rev Bras Otorrinolaringol. 2005; 71(6):747-51.

12. Degan VV, Puppin-Rontani RM. Prevalence of pacifier-sucking habits and successful methods to eliminate them: a preliminary study. J Dent Child. 2004 May-Aug; 71(2):148-51.

13. Viggiano D, Fasano D, Monaco G, Strohmenger L. Breast feeding, bottle feeding and non-nutritive sucking: effects on occlusion in deciduous dentition. Arch Dis Child. 2004; 89(12):1121-3.

RECEBIDO EM: 10/10/2008

ACEITO EM: 09/09/2009

Endereço para correspondência:

Joanna Carolina Bachiega

Estrada do Cascalho, viela 1, 51, casa 3

São Sebastião - SP

CEP: 11600-000

E-mail: jocarolina@ig.com.br
14. Leite-Cavalcanti A, Medeiros-Bezerra PK, Moura C. Aleitamento natural, aleitamento artificial, hábitos de sucção e maloclusões em pré-escolares brasileiros. Rev Salud Pública. 2007; 9(2):194-204. 15. Bayardo RA, Peixoto LFS, Corrêa MSNP. Aleitamento natural e artificial: considerações gerais. J Bras Clin Odontol Integr. 2003; 7(39):257-60.

16. Chase WR. Imperative early treatment of anterior open bite. Gen Dent. 1993; 41(4):307-9.

17. Ovsenik M, Farcnik FM, Verdenik I. Comparison of intra-oral and study cast measurements in the assessment of malocclusion. Eur J Orthod. 2004; 26(3):273-7.

18. Silva EL. Hábitos bucais deletérios. Rev Para Med. 2006 jun; 20(2):47-50.

19. Harila V, Heikkinen T, Gron M, Alvesalo L. Open bite in prematurely born children. J Dent Child. 2007 Set-Dec; 74(3):165-70.

20. Li JN, Kanno ZS, Soma K. [Study of occlusal force on female open bite patients with temporomandibular disorders]. Hua Xi Kou Qiang Yi Xue Za Zhi. 2007 Dec; 25(6):536-9.

21. Cattoni DM, Fernandes FDM, Di Francesco RC, Latorre MRDO. Características do sistema estomatognático de crianças respiradoras orais: enfoque antroposcópico. Pró-Fono. 2007 out-dez; 19(4):347-51.

22. Rosell Puche L. Mordida abierta: diagnóstico y tratamiento. Acta Odontol Venez. 1991 May-Ago; 29(2):53-60.

23. Zuanon ACC, Oliveira MF, Giro EMA, Maia JP. Relação entre hábito bucal e maloclusão na dentadura decídua. J Bras Odontopediatr Odontol Bebê. 2000; 3(12):104-8.

24. Gois EGO, Ribeiro Júnior HC, Vale MPP, Paiva SM, Serra-Negra JMC, Ramos-Jorge ML, et al. Influence of nonnutritive sucking habits, breathing pattern and adenoid size on the development of malocclusion. Angle Orthod. 2008 Jul; 78(4):647-54. 25. Degan VV, Puppin-Rontani RM. Remoção de hábitos e terapia miofuncional: restabelecimento da deglutição e repouso lingual. Pró-Fono. 2005; 17(3):375-82.

26. Degan VV, Puppin-Rontani RM. Aumento da aeração nasal após remoção de hábitos de sucção e terapia miofuncional. Rev CEFAC. 2007 jan-mar; 9(1):55-60. dx.doi.org/10.1590/ S1516-18462007000100008

27. Jefferson Y. Orthodontic diagnosis in young children: beyond dental malocclusions. Gen Dent. 2003; 51(2):104-11.

28. Laucis-Pinto $S$, Diegues MB, Ferreira SLM, Simonato CASS. Bruxismo em odontopediatria e sua correlação com hábitos orais. Rev Paul Odontol. 2000 set-out; 22(5):10-8. 